\title{
Colonoscopic findings in Crohn's disease - Reproducible, but of questionable benefit
}

\author{
H HOLLOWAY MD, JF FIELDING FACG
}

\begin{abstract}
H Holloway, JF FieldiNG. Colonoscopic findings in Crohn's disease - Reproducible, but of questionable benefit. Can J Gastroenterol 1996;10(6):405-412. Colonoscopy, a useful diagnostic tool in inflammatory bowel disease, is very accurate in detecting disease and assessing disease extent in Crohn's disease. Despite their accuracy, colonoscopic findings have not been routinely used in the objective follow-up of patients with Crohn's disease. The GETAID (Groupe d'études theraputic des affections inflammatoire du tube digestif) group has shown that following an intensive training period, endoscopists can describe colonoscopic findings in a reproducible manner. However, these descriptions poorly correlate both with clinical or laboratory indexes, and with short or medium term prognosis following prednisolone therapy.
\end{abstract}

Key Words: Colonoscopy, Crohn's disease, GETAID

\section{Coloscopie dans la maladie de Crohn : Résultats reproductibles, mais d'une utilité douteuse}

RÉSUMÉ : La coloscopie, un outil diagnostique utile dans la maladie inflammatoire de l'intestin, est très précise lorsqu'il s'agit de déceler et d'évaluer l'étendue de la maladie de Crohn. Malgré leurs précisions, les résultats de la coloscopie n'ont toutefois pas été utilisés d'emblée pour le suivi objectif des patients atteints de la maladie. Le GETAID (Groupe d'études thérapeutiques des affections inflammatoires du tube digestif) a démontré qu'après une période de formation intensive, les médecins qui pratiquent l'endoscopie peuvent décrire des résultats de coloscopie de façon reproductible. Toutefois, ces descriptions sont en piètre corrélation avec les indices cliniques ou les indices des analyses de laboratoire et avec le pronostic à court ou à moyen terme après un traitement par prednisolone.
$T^{1}$ he introduction of fibreoptic and video endoscopy significantly altered the diagnosis and understanding of gastrointestinal complaints. Endoscopy is now considered essential for the objective evaluation of drug efficacy in many upper gastrointestinal diseases.

Colonoscopy allows direct visualization of colonic mucosa and the opportunity to collect multiple biopsy specimens. Studies have shown that colonoscopy with biopsy is superior to radiology alone in the diagnosis of Crohn's colitis, particularly when mucosal changes are mild (1); is helpful in the differential diagnosis between ulcerative colitis and colonic Crohn's disease (2); and provides a more precise delineation of disease extent than radiology (3). Nonetheless, and in sharp contrast to the prominent role played by endoscopy in the diagnosis and management of other digestive diseases, endoscopic findings have not yet been routinely used in the objective follow-up of patients with Crohn's disease.

Several indexes have been proposed for the assessment of drug efficacy in Crohn's disease (4-7). These indexes rely mainly on subjective symptoms, objective laboratory parameters or both. Endoscopic lesions, although well known, vary from patient to patient. The nature, site, severity or extent of macroscopic disease at endoscopy has only recently been correlated with disease activity (8-10).

This article reviews the current state of colonoscopy in relation to the presence, extent and activity of Crohn's colitis, and to the follow-up of patients with Crohn's colitis to determine whether endoscopic findings can be incorporated in the assessment of disease activity.

GETAID (Groupe d'études theraputic des affections in-

Royal College of Surgeons in Ireland and Beaumont Hospital, Dublin, Ireland

Correspondence and reprints: Professor JF Fielding, Department of Medicine, Mater Private Hospital, Dublin 7, Ireland. Telephone 3531

8300808, fax 35318300810

Received for publication February 9, 1995. Accepted September 29, 1995 


\section{TABLE 1}

Nine elementary mucosal lesions

\begin{tabular}{|c|c|}
\hline Lesion & Definitions or specifications \\
\hline \multicolumn{2}{|l|}{ Pseudopolyp } \\
\hline Healed ulceration & $\begin{array}{l}\text { Whitish area with a 'ground glass' } \\
\text { appearance }\end{array}$ \\
\hline $\begin{array}{l}\text { Frank erythema } \\
\text { (plaques, bands or } \\
\text { diffuse) }\end{array}$ & $\begin{array}{l}\text { Slight or moderate erythema should } \\
\text { be neglected }\end{array}$ \\
\hline Frankly swollen mucosa & $\begin{array}{l}\text { Slight or moderate mucosal swelling } \\
\text { should be neglected }\end{array}$ \\
\hline Aphthoid ulceration & $\begin{array}{l}\text { A tiny }(2-3 \mathrm{~mm}) \text { raised or flat red } \\
\text { lesion with a white centre }\end{array}$ \\
\hline $\begin{array}{l}\text { Superficial or shallow } \\
\text { ulceration }\end{array}$ & $\begin{array}{l}\text { Any ulceration that is neither aphthoid } \\
\text { nor deep }\end{array}$ \\
\hline Deep ulceration & Only frankly deep ulcerations \\
\hline Nonulcerated stenosis & $\begin{array}{c}\text { Should be impossible or difficult to } \\
\text { pass with an adult endoscope }\end{array}$ \\
\hline Ulcerated stenosis & $\begin{array}{l}\text { Should be impossible or difficult to } \\
\text { pass with an adult endoscope }\end{array}$ \\
\hline
\end{tabular}

flammatoire du tube digestif), a French group composed of gastroenterologists and endoscopists from 12 university centres, was the first to acknowledge the lack of endoscopic data in controlled clinical trials. They performed a series of prospective studies that investigated the definition and reproducibility of colonoscopic findings in Crohn's disease (11); the development and validation of an endoscopic index of severity (12); the relationship among clinical, biological and endoscopic parameters in Crohn's disease (8); and the use of endoscopic monitoring of Crohn's disease treatment (13).

\section{ENDOSCOPIC FINDINGS: DEFINITION AND REPRODUCIBILITY OF MUCOSAL LESIONS}

The main aim of GETAID's first study was to demonstrate whether a multicentre group could standardize data collection and achieve a reasonable level of agreed colonoscopic findings in Crohn's disease (11). The study group reached agreement on the definition of the nine mucosal lesions whose presence or absence was to be recorded (Table 1). These nine lesions were not correlated with disease activity, although the Crohn's Disease Activity Index (CDAI) had been calculated in the majority of patients studied.

A total of 112 colonoscopies were performed by 18 intracentre endoscopist pairs (both from the same centre). A further 21 colonoscopies were performed by 14 intercentre endoscopist pairs (each from a different centre). Patients with colonic or ileocolonic disease were included. Patients who had undergone previous proctocolectomy or total colectomy with ileorectal anastomosis were excluded. The study group failed to state whether patients with previous segmental resections were included.

Endoscopic information collected during each colonoscopy included the following: presence or absence of the nine mucosal lesions for each of five colonic segments (rectum, sigmoid and left colon, transverse colon, right colon and ileum); percentage of segmental surfaces involved by disease (any of the nine mucosal lesions) (SSD); and percentage of segmental surfaces involved by ulcerations only (SSU).

After recording the segmental data described, endoscopists were required to record independently their global estimate of lesion severity (GELS) on a five-point scale (healed, minor, moderate, severe or very severe). GELS was subsequently used as the dependent variable in the development of a Crohn's disease endoscopic index of severity.

The reproducibility of colonoscopic findings was based on observer agreement or disagreement on the presence or absence of the nine mucosal lesions in the protocol (Table 2).

Interobserver variation for lesion detection was estimated by the calculation on agreement on positive $[\mathrm{Po}(+)]$ and negative $[\mathrm{Po}(-)]$ findings using $2 \times 2$ contingency tables. $\mathrm{Po}(+)$ and $\mathrm{Po}(-)$ are simple measures of observer variations and are not adjusted for agreement that can be expected by chance alone. The expected chance agreement $(\mathrm{Pc})$ is $\mathrm{Po}(+)^{2}+\mathrm{Po}(-)^{2}$. The definition of the Kappa coefficient is $(\mathrm{Po}-\mathrm{Pc}) /(1-\mathrm{Pc})$. Kappa values can assume values between -1 (complete disagreement) and +1 (complete agreement). Values around zero indicate chance agreement alone.

Agreement on positive findings was uniformly good (for both the intra- and intercentre studies) for the following mucosal lesions: pseudopolyps, superficial and deep ulcera-

TABLE 2

Global agreement on positive $[\mathrm{Po}(+)]$ and negative $[\mathrm{Po}(-)]$ findings and Kappa levels concerning detection of elementary lesions

\begin{tabular}{|c|c|c|c|c|c|c|c|c|}
\hline & \multicolumn{4}{|c|}{ Intracentre study (n=112) } & \multicolumn{4}{|c|}{ Intercentre study $(\mathrm{n}=21)$} \\
\hline & $\mathrm{Po}(+)$ & Po(-) & Kappa & $\mathbf{P}<$ & $\mathrm{Po}(+)$ & Po(-) & Kappa & $\mathbf{P}<$ \\
\hline Pseudopolyp & 0.92 & 0.96 & 0.88 & 0.001 & 0.80 & 0.93 & 0.73 & 0.01 \\
\hline Healed ulceration & 0.79 & 0.97 & 0.76 & 0.001 & 0.57 & 0.97 & 0.54 & 0.05 \\
\hline Frankly swollen mucosa & 0.76 & 0.94 & 0.70 & 0.001 & 0.43 & 0.92 & 0.36 & NS \\
\hline Aphthoid ulceration & 0.88 & 0.98 & 0.85 & 0.001 & 0.67 & 0.95 & 0.62 & 0.05 \\
\hline Nonulcerated stenosis & 1 & 1 & 1 & 0.001 & * & * & * & * \\
\hline Ulcerated stenosis & 0.93 & 0.99 & 0.93 & 0.001 & 1 & 1 & 1 & 0.001 \\
\hline
\end{tabular}

*No calculation could be performed for this item in the intercentre study because it was seen in only one patient. NS Not significant 
TABLE 3

Format for calculation of the Crohn's Disease Endoscopic Index of Severity (CDEIS)

\begin{tabular}{|c|c|c|c|c|c|c|}
\hline Sample colonoscopy & Rectum* & $\begin{array}{c}\text { Sigmoid and } \\
\text { left colon }\end{array}$ & $\begin{array}{c}\text { Transverse } \\
\text { colon }^{\ddagger}\end{array}$ & $\begin{array}{l}\text { Right } \\
\text { colon } \$\end{array}$ & lleum $\llbracket$ & Row total \\
\hline $\begin{array}{l}\text { Deep ulceration } \\
\text { quote } 12 \text { if present in the segment } \\
\text { quote } 0 \text { if absent }\end{array}$ & 0 & 0 & 12 & 0 & - & 12 (Total 1) \\
\hline $\begin{array}{l}\text { Superficial ulceration } \\
\text { quote } 6 \text { if present in the segment } \\
\text { quote } 0 \text { if absent }\end{array}$ & 0 & 0 & 6 & 6 & - & 12 (Total 2) \\
\hline Ulcerated surface (measured in $\mathrm{cm})^{\star}$ & 0 & 0 & 6 & 1.5 & - & 7.5 (Total 4) \\
\hline \multicolumn{6}{|c|}{ Total $1+$ Total $2+$ Total $3+$ Total 4} & 47.5 (Total A) \\
\hline \multicolumn{6}{|c|}{ Number of segments totally or partially explored (1.5) } & $4(n)$ \\
\hline \multicolumn{6}{|c|}{ Total A divided by $n$} & 11.9 (Total B) \\
\hline
\end{tabular}

For partially explored segments and for the ileum, the $10 \mathrm{~cm}$ linear scale represents the surface effectively explored. The findings at this sample colonoscopy are as follows: *Normal rectum; ${ }^{\dagger}$ Presence of nonulcerative lesions involving $20 \%$ of the sigmoid and left colon area; ${ }^{\ddagger}$ Disease affecting $80 \%$ of the transverse colon area including superficial and deep ulcerations, with ulcerations representing $60 \%$ of the segment surface; $\$$ Incomplete exploration due to a nonulcerated stenosis; $60 \%$ of the explored right colon was diseased, $15 \%$ accounted for by superficial ulcerations; "Ileum was not reached

tions, and ulcerated and nonulcerated stenosis. At least $75 \%$ of endoscopists had Kappa values above 0.80 for all the above lesions.

As noted in Table 2, healed ulcerations and aphthoid ulcerations showed good intracentre reproducibility but lower intercentre reproducibly; 'frank' erythema and 'frankly' swollen mucosa demonstrated reasonable intracentre observer agreement but poor intercentre reproducibility.

Observer agreement in the evaluation of SSD and SSU was surprisingly good despite the great subjectivity inherent in such evaluations. Global values of the interobservers coefficients were 0.85 and 0.88 in the intracentre, and 0.88 and 0.73 in the intercentre studies for SSD and SSU, respectively $(\mathrm{P}<0.001$ in all cases $)$.

GELS was estimated independently by both endoscopists on a five-point scale of severity (healed $=1$, minor $=2$, moderate $=3$, severe $=4$, very severe $=5$ ). Complete agreement was achieved in 81 of 112 intracentre colonoscopies (72.3\%) and 14 of 21 intercentre colonoscopies (66.7\%) (not significant). A further 29 (26\%) of the intracentre colonoscopies were agreed to within one scale of the fivepoint scale, as were five $(24 \%)$ of the intercentre study. Larger discrepancies in the GELS by the endoscopist pair were due to the divergent interpretation of healed ulcerations; one endoscopist considered ulcers with re-epithelialization to be healed, while the coinvestigator considered ulcers with re-epithelialization that was not flush with adjacent margins to have either moderate (intracentre) or severe (intercentre) disease.

As can be seen from Table 2, there was a trend towards better reproducibility of colonoscopic findings in the intracentre studies. Most endoscopic lesions were better recorded in the intracentre study, except for superficial ulcerations and ulcerated stenosis which were recorded with equal reproducibility by both the intracentre and intercentre studies $(\mathrm{P}<0.001$ for both).

The lesser reproducibility of intercentre endoscopic findings possibly would have reached statistical significance if there had been more intercentre colonoscopies. The reproducibility of colonoscopic findings between endoscopists not involved in the GETAID study is unknown but is unlikely to be as good as even the intercentre group. This point was alluded to by the GETAID group in their discussion; they stated that their training period was essential and that endoscopic reproducibility was not immediately 'transposable' elsewhere.

Following GETAID's study of the feasibility of a cooperative multicentre group to collect endoscopic data in a standardized fashion, the GETAID group developed and validated the first endoscopic index of severity in Crohn's disease: the Crohn's Disease Endoscopic Index of Severity (CDEIS) (Table 3).

Endoscopic findings were prospectively recorded by a multicentre group in 75 patients with Crohn's colitis using the standardized protocol of the previous study. Presence or absence of the nine preselected mucosal lesions was recorded for each of the five colonic segments examined (rectum, sigmoid and left colon, transverse colon, right colon and ileum). SSD and SSU were also recorded. These independent variables were recorded together with the dependent variable, the endoscopist GELS.

Stepwise multiple regression analysis was used to derive an index from the independent variables that best correlated with the dependent variable. Four mucosal lesions - superficial ulcerations, deep ulcerations, and ulcerated and nonulcerated stenosis - together with the estimated extent involved were selected and weighted to obtain the CDEIS that correlated best with the endoscopists' GELS ( $r=0.83$, 


\section{TABLE 4 \\ Major and minor points used to confirm diagnosis of Crohn's disease}

\section{Major points}

Fissure, ie, cleft-like ulcerations

Interspersed normal and pathological findings

Minor points

Aphthous lesions with normal-appearing mucosa

Anal lesions (fistulas, chronic fissures)

Rectum spared but involvement of the colon

$\mathrm{P}<0.001)$. The index was prospectively validated in a further series of 103 colonoscopies $(r=0.81, P<0.001)$.

The CDEIS relies on precise data that may limit its use in clinical practice. If an endoscopic index can be used in therapeutic trials it should be sufficiently simple to be acceptable and capable of accurate compilation on a wide basis. The variables incorporated in such an endoscopic index should reflect disease activity rather than disease extent.

The CDEIS comprises mainly graded variables such as the depth and extent of ulcerations. Ulcerations may indeed reflect active inflammation; however, it is difficult, if not impossible, to decide whether ulcers are increasing or decreasing in depth, or are undergoing epithelialization, by macroscopic appearance alone.

The use of graded or continuous variables was shown to lack precision in a previous study (11). These workers stated that characteristics that could be used with some confidence in describing mucosal appearances in proctocolitis were those for which observers recorded the presence or absence (discontinuous) of mucosal lesions rather than the use of graded variables (continuous) (14).

Widespread use of the CDEIS may be limited due to its complexity. What is needed in multicentre trials is a simple index that incorporates the presence or absence of mucosal lesions and is capable of clear definition. Ideally such a simple index also reflects disease activity.

\section{ENDOSCOPIC FINDINGS AND THEIR CORRELATION WITH CLINICAL INDEXES OR LABORATORY PARAMETERS OF ACTIVITY}

If an endoscopic index is to be used in therapeutic trials, it should incorporate variables reflecting disease activity. Most of the typical Crohn's lesions detectable by endoscopy appear to be well defined. The clinical relevance and prognostic implications of the appearance, site and extent of such endoscopic lesions have not been extensively studied. Limited studies $(8-10)$ have demonstrated a poor correlation between endoscopic parameters and clinical indexes or symptoms.

The European Cooperative Crohn's Disease Study Group (ECCDS) (9) observed colonoscopic findings in $130 \mathrm{pa}$ tients and published their results in 1985 . The group attempted to elucidate the clinical relevance of mucosal lesions in Crohn's disease by studying the correlation of the extent and frequency of mucosal lesions with the following: a clinical index of activity (CDAI); the duration of symptoms; and time since confirmation of diagnosis.

Diagnosis of Crohn's disease was confirmed on an endoscopic basis when at least two major points or a combination of at least one major and two minor points were present (Table 4).

The rectum and sigmoid colon were examined in all 130 patients in the ECCDS study. The distal part of the descending colon to the midtransverse colon was investigated in 98 patients ( $75 \%$ ) and the cecum was seen in $66(51 \%)$. Inspection of the terminal ileum was achieved in 21 of the cases $(16 \%)$ (this figure was taken from table 3 of their text [9] and does not correspond to the "nearly" $10 \%$ quoted in the results section).

The findings of the ECCDS study must be open to some doubt. First, pathological findings were not observed in 17 of the 130 patients $(13 \%)$. Thirteen of the 17 patients had complete colonoscopy and three had at least moderately active disease, judged by a CDAI of greater than 250. Why did the authors include these patients, who did not conform to the endoscopic criteria for inclusion? How could some patients have active disease in the absence of abnormal findings on a "completely inspected" examination, or were they ileocolonic patients with only small bowel activity at that point?

Second, a segmental pattern of Crohn's disease involvement was seen in $86 \%$ of patients. Fourteen per cent had evidence of continuous Crohn's disease. No correlation was established between disease extent on endoscopy and CDAI at a given point.

Next, 33 patients (25\%) had previous resections, mostly involving the ileum and right side of the colon. The anastomosis was inspected in 23 patients, 17 of whom (74\%) showed signs of macroscopic recurrence at or near the anastomosis. Stenosis and ulcerations were the dominant forms of mucosal lesions followed by 'aphthous ulcers' and erosions. Overall, clinical activity did not differ significantly between patients with and without previous surgical resections. However, 10 of the 17 patients had only "minor activity" which the authors described as a CDAI score of less than 150. Five of the seven patients with "more active" disease and endoscopic recurrence showed evidence of local stenosis, suggesting that stenosis, rather than active disease, may have accounted for their symptoms. This may reflect the symptomatic nature of the CDAI rather than necessarily reflecting a correlation or noncorrelation between their endoscopic findings and disease activity.

Fourth, the presence of ulcers appeared to be associated with symptoms (indirectly confirmed by CDAI). Symptoms usually lead patients to visit a doctor, and symptomatic patients are therefore more likely to be endoscoped. This may explain why the ECCDS group noted that ulcers occurred more frequently in patients endoscoped shortly after presenting with symptoms (short time since confirmation of diagnosis) but that ulcerations occurred at all times of disease duration. Relatively more asymptomatic patients are more likely to be endoscoped during follow-up. 
Finally, cobblestone lesions and pseudopolyps were associated with a short period of symptomatology and did not correlate with a short time since diagnosis confirmation. The ECCDS group stated that these lesions may be "early signs of Crohn's colitis". This may be an erroneous deduction by the authors. It is well known that the characteristic cobblestone mucosa seen in Crohn's disease is a sign of advanced or well established disease (15). Symptoms may have occurred relatively late in the disease process and thus these lesions may have been erroneously labelled as early lesions.

In 1986 Gomes and co-workers (10) published their study on the relationship between disease activity indexes and colonoscopic findings in patients with colonic inflammatory bowel disease (IBD). They noted that increasing reliance was being placed on subjective clinical indexes and/or laboratory parameters in therapeutic trials in Crohn's disease. Gomes et al attempted to discover how well these nonspecific parameters of activity related to disease extent and severity (macroscopic and microscopic scores) in patients with colonic IBD.

Colonoscopic findings of 28 patients with ulcerative colitis and 22 patients with Crohn's colitis were studied. Before each colonoscopy the clinical activity indexes, the CDAI and the more simplified Harvey and Bradshaw version (simple index of activity), were calculated for each patient with Crohn's disease. The group failed to state whether each patient was required to fill in a diary card as demanded by the original CDAI or the study relied solely on patient recall. Before each colonoscopy, blood was taken for full blood count, platelet count, and albumin and $\mathrm{C}$ reactive protein levels. At colonoscopy the colon was divided into six notional sections defined as the cecal area, hepatic flexure area, splenic flexure area, descending colon, sigmoid colon and rectum. Each section was assessed for the presence or absence of macroscopic disease by one of three experienced colonoscopists on a scale of 0 to 3 (Table 5). The histological extent and activity of the disease, graded on a scale of 0 to 4 (Table 5), was assessed by a single histopathologist with a special interest in gastrointestinal disease. The pathologist was unaware of the clinical status and endoscopic findings of the patients.

Overall, Gomes and associates found a poor correlation between colonoscopic or histological findings and clinical activity indexes. However, their results may be misleading. First, only 22 patients with Crohn's disease were studied. Second, the macroscopic and microscopic parameters used in their scoring systems were not clearly defined. Third, many patients were on medical treatment at entry to the study. Such medical intervention may have provided symptomatic relief with less or even no accompanying beneficial effect on endoscopic or histological findings. Finally, the macroscopic and microscopic parameters used by single observers reflected disease activity more accurately for patients with ulcerative colitis rather than for those with Crohn's disease.

Despite these limitations there was still a significant, but not close, correlation between the macroscopic score and the

\section{TABLE 5}

\section{Scoring system}

\begin{tabular}{lc}
\hline Macroscopic score \\
0 & Normal \\
1 & Mild inflammation with loss of vascular pattern with or without \\
granularity or localized aphthous ulcers \\
2 & Severe inflammation with contact bleeding \\
3 & $\begin{array}{c}\text { More severe disease with friability, ulcers or spontaneous } \\
\text { bleeding }\end{array}$ \\
Microscopic score \\
$0 \quad$ Normal \\
1 & Mild edema and inflammation in the lamina propria \\
2 & Crypt abscess formation and inflammation in the lamina \\
3 & propria \\
& More severe inflammation with destructive crypt abscesses \\
4 & More severe inflammation with active ulceration \\
\hline
\end{tabular}

simple index of activity in patients with Crohn's colitis $(\mathrm{r}=0.68, \mathrm{P}<0.05)$. However, the simple index alone may not be an accurate assessor of disease activity (16).

In Crohn's colitis, the macroscopic appearance tended to underestimate the histological extent of disease, but there was a good correlation between microscopic and macroscopic scores $(\mathrm{r}=0.76, \mathrm{P}<0.001)$. There was, however, no correlation between microscopic score and simple index of activity.

Moreover, no correlation was noted between either the microscopic or macroscopic score and any of the laboratory parameters measured ( $\mathrm{C}$ reactive protein, albumin, hemoglobin, erythrocyte sedimentation rate and platelets).

In both ulcerative colitis and Crohn's colitis there was no significant correlation between maximum area of severity (either macroscopically or microscopically) and clinical or laboratory indexes of activity. At any point, 'random' histology (based on minute biopsies) is limited in Crohn's disease; the reliability of single segmental samples to assess disease extent and activity in Crohn's disease, which is often of patchy distribution and severity, must be questioned.

The GETAID workers may have been the first group to develop a standardized, reproducible method of recording endoscopic data but, as has been noted, were not the first to attempt to correlate clinical and endoscopic findings. Indeed, they only attempted such a correlation during the first practical application of their systematic approach to colonoscopy.

The GETAID group subsequently undertook a multicentre prospective trial; its main aim was to assess the value of endoscopic follow-up for adjusting the dose and duration of steroid treatment in patients with active Crohn's disease. Thirteen clinical, three biological and 12 endoscopic parameters were collected from 142 patients with active colonic disease (CDAI at least 200) before and after a three- to seven-week course of oral prednisolone (Table 6). These data formed the basis of their third publication, in 1990, on the clinical, biological and endoscopic features in Crohn's disease (10). 
TABLE 6

List of the 28 variables collected at entry

\begin{tabular}{l} 
Clinical variables ( $\mathbf{n} \mathbf{1 3}$ ) \\
Age at Crohn's disease onset \\
Age at day 0 of the trial \\
Time elapsed between Crohn's disease onset and diagnosis \\
Time elapsed between Crohn's disease onset and day 0 of the trial \\
Time elapsed between diagnosis and day 0 of the trial \\
First attack \\
Previous surgical treatment \\
Previous medical treatment \\
Medical treatment in the month preceding day 0 of the trial \\
Small bowel disease \\
Anal lesions \\
Crohn's Disease Activity Index \\
Biological variables ( $\mathbf{n}=\mathbf{3}$ ) \\
Hemoglobin \\
Erythrocyte sedimentation rate \\
Serum albumin \\
Endoscopic variables ( $\mathbf{n}=12$ ) \\
Average segmental surface involved by Crohn's disease \\
(any lesion) \\
Average segmental surface involved by ulcerations \\
Individual segmental rectocolonic frequency of the nine \\
elementary mucosal lesions \\
Crohn's Disease Endoscopic Index of Severity \\
\hline
\end{tabular}

In that study, clinical and endoscopic parameters were studied in 55 patients (39\%) with ileocolonic disease and 57 patients $(40 \%)$ with colonic disease. No correlation between the CDAI and the CDEIS was observed at study entry $(r=0.13)$. This lack of correlation between indexes was also found for patients with colonic disease only $(\mathrm{r}=0.15)$.

To study the relationship between mucosal lesions and clinical parameters patients with active disease (CDAI at least 200) were divided into subgroups of increasing frequency of weekly stool scores ( 0 to $14 ; 15$ to $35 ; 36$ to 56 ; greater than 56$)$, pain scores ( 0 to $7 ; 8$ to 14 ; greater than 14$)$ and CDAI values (200 to 300; 301 to 400; greater than 400). The individual segmental rectocolonic frequency (ISRCF) the ratio of the number of segments showing a given lesion to the number of colonic segments examined in a given patient (calculated for all nine mucosal lesions) - was correlated with the clinical parameters described above. There was a poor correlation of both symptoms and index (CDAI) with mucosal parameters as calculated by the ISRCF.

Increasing stool frequency, pain or CDAI scores were not associated with any mucosal lesion. The only significant finding was that patients with a normal ileum had slightly but significantly $(\mathrm{P}<0.05)$ lower pain scores than patients with diseased ileum. The ilea have a small circumference; therefore patients with diseased ilea are more likely to suffer obstructive symptoms from stenosis as previously noted (9).

An average of segmental surface involved by disease (ASSD) and by ulcerations only (ASSU) was calculated by dividing the sum of the SSD and SSU by the number of segments examined. No correlation was found between the CDAI and lesion surface $(r=0.04)$ or ulcerated surface $(\mathrm{r}=0.15)$.

Like previous researchers, the GETAID group failed to find a correlation between clinical and endoscopic severity. However, unlike their predecessors, they studied patients according to macroscopic location (colonic and ileocolonic). Different clinical patterns are associated with different areas of macroscopic involvement (17). If the presence of macroscopic disease and the frequency, extent and depth of mucosal lesions in the three major subgroups (ileal, ileocolonic and colonic) were studied, then - provided patients within each subgroup were as homogeneous as possible (eg, regarding age, disease duration and previous surgical history) perhaps a closer correlation between clinical and endoscopic data would be achieved.

There was no correlation between the CDEIS and hemoglobin concentrations or erythrocyte sedimentation rates. The GETAID group noted a significant negative correlation between serum albumin and the CDEIS $(\mathrm{r}=-0.31, \mathrm{P}<0.01)$. Active Crohn's biological inflammation implies the presence of exudative lesions; $67 \%$ of the variables used in the endoscopic index relate to the presence or absence of ulcerative lesions. Such lesions can lead to excessive protein loss and hypoalbuminemia. In a previous study Cooke and Prior (18) demonstrated that seromucoids were significantly inversely correlated with serum albumin levels and reflected disease activity more accurately than either erythrocyte sedimentation rates or hemoglobin concentrations. Therefore the CDEIS may have reflected endoscopic disease activity because it had a significant negative correlation with a more widely agreed upon laboratory parameter of disease activity (16).

However, before an endoscopic index can be accepted in therapeutic trials or provide a more objective long term follow-up of patients, further studies using a simple index on subgroups of patients will be required.

Despite the lack of correlation between clinical activity and any of the endoscopic data (lesion frequency, surface area involved or endoscopic index of severity), the GETAID group used their endoscopic index (CDEIS) in a randomized clinical trial (13).

\section{ENDOSCOPIC MONITORING OF CROHN'S DISEASE TREATMENT}

Medical follow-up: The main aim of the GETAID clinical trial was to determine the prognostic value of colonoscopy in patients achieving clinical remission after a high dose of prednisolone. Included in the trial were 147 patients with acute attacks of ileocolonic or colonic Crohn's disease (CDAI greater than 200 or at least 200). Each patient was treated with oral prednisolone $1 \mathrm{mg} / \mathrm{kg} /$ day over three to seven weeks to induce clinical remission (CDAI less than 150 with a decrease of at least 100 below the value of entry).

Clinical remission was achieved in 136 patients (93\%). Forty of these patients (27\%) (group C) were also in endo- 


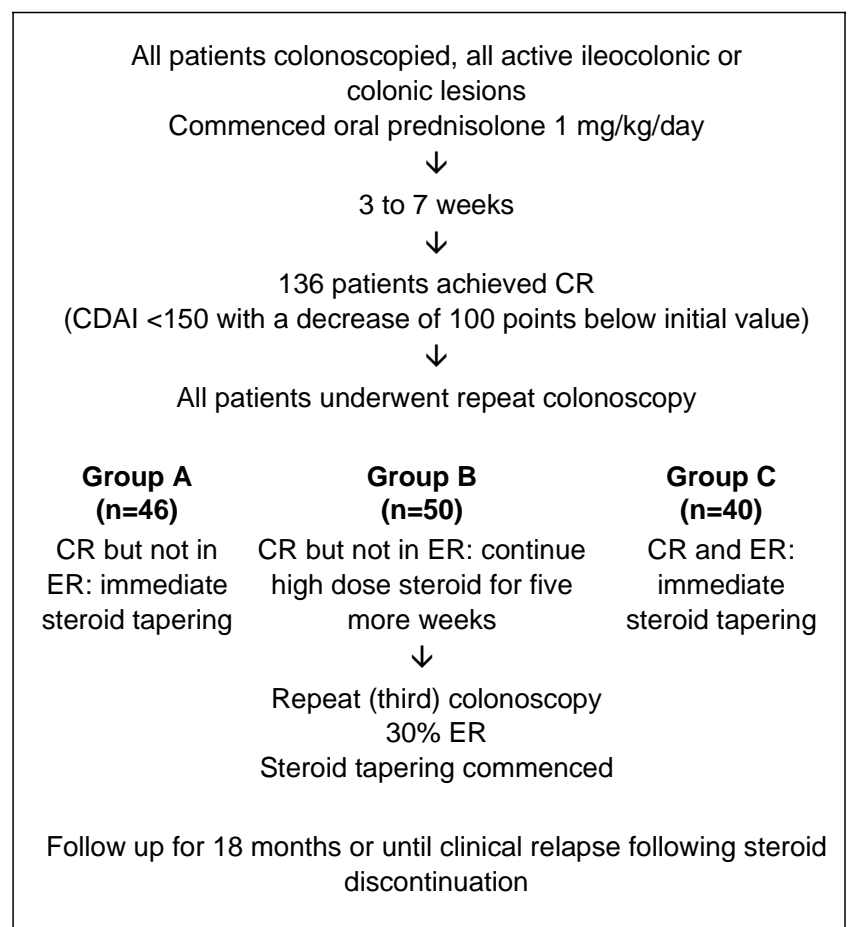

Figure 1) GETAID (Groupe d'études theraputic des affections inflammatoire du tube digestif) clinical trial (13): 147 patients with acute attacks of ileocolonic or colonic Crohn's disease (Crohn's Disease Activity Index [CDAI] at least 200) were included. CR Clinical remission; ER Endoscopic remission

scopic remission (ER), defined as no lesions or scarred lesions only (full ER); or minor lesions with at least a two-grade decrease on a six-grade scale of endoscopic severity with no residual deep ulceration compared with previous colonoscopic findings (partial ER). The remaining 96 patients $(71 \%)$ still had active endoscopic lesions; they were randomly assigned either to start steroid tapering immediately ( $n=46$, group A) or to prolong prednisolone therapy at the same dosage for a further five weeks before tapering was begun ( $n=50$, group $B$ ). Colonoscopy was performed again in group $\mathrm{B}$ after this prolonged therapy. In the remaining 40 patients with both clinical and ER (group C), steroid therapy was also tapered immediately (Figure 1).

Following prolongation of steroid therapy in group B, 32 of the 50 patients $(64 \%)$ had an overall improvement of endoscopic lesions. However, only 15 patients (30\%) achieved full (12) or partial (three) ER. Although prolongation of prednisolone treatment in group $\mathrm{B}$ was associated with a significant endoscopic improvement $(\mathrm{P}<0.01)$, it did not appear to influence clinical outcome according to the following judgement criteria used by the study group.

The first criterion was that there was no difference among the percentage of patients (in groups A, B and C) who could be weaned successfully off steroid treatment while maintaining clinical remission over the 18 months; 40 patients ( $87 \%)$ in group A, 37 (74\%) in group B and $36(90 \%)$ in group C were successfully weaned off steroids.

The second criterion was that, because prolonged pred-

\section{TABLE 7}

\section{Summary of results}

\section{ECCDS study (9): Colonoscopy}

1 No correlation between CDAI and disease extent

2 No significant difference in clinical activity between patients with and without previous resections

3 Patients with ulcerations had short time since confirmation of diagnosis

\section{Gomes et al (10)}

1 Significant correlation between Harvey and Bradshaw Index and macroscopic score $(r=0.76, P<0.001)$

2 No correlation between Harvey and Bradshaw Index and microscopic score

3 No correlation between either macroscopic or microscopic scores and each of the following laboratory parameters: hemoglobin, ESR, platelets, albumin, $C$ reactive protein

\section{GETAID (8,11-13)}

1 No correlation between CDAI and CDEIS for ileocolonic or colonic only Crohn's disease

2 Poor correlation among stool frequency, pain scores, CDAI with frequency of nine mucosal lesions, ASSD and ASSU

3 Significant correlation between disease ilea and pain $(P<0.05)$

4 No correlation among the CDEIS, hemoglobin and ESR

5 Significant correlation between the CDEIS and albumin $(r=-0.31, P<0.01)$

6 Endoscopic data collected before steroid tapering had no predictive value on either the short term (up to seven weeks) or medium term (up to 18 months) prognosis in acute attacks of Crohn's disease treated with oral prednisolone

Rutgeerts et al (15)

1 Endoscopy identifies postoperative recurrences

2 The severity of the postoperative recurrence predicts the speed of clinical recurrence

ASSD Average of segmental surface involved by disease; ASSU Average of segmental surface involved by ulcerations only; CDAl Crohn's Disease Activity Index; CDEIS Crohn's Disease Endoscopic Index of Severity; ECCDS European Cooperative Crohn's Disease Study Group; ESR Erythrocyte sedimentation rate; GETAID Groupe d'études theraputic des affections inflammatoire du tube digestif

nisolone therapy did not appear to influence the relapse rate (group B), the researchers also compared the clinical outcome of all patients in ER before steroid tapering (all of group $\mathrm{C}$ and group $\mathrm{B}$ in ER following prolongation of therapy) with that of patients who retained endoscopic lesions (all of group A and group B not in ER after prolongation of therapy). Forty-six of 52 patients in ER (88\%) compared with 64 of 80 patients not in ER (80\%) were weaned off prednisolone $(\mathrm{P}>0.2)$. The relapse rate after steroid withdrawal was similar in both groups $(\mathrm{p}=0.68)$.

Based on the above data, endoscopic data collected before steroid tapering had no predictive value on either immediate clinical response or subsequent clinical outcome. Endoscopy, therefore, was of no benefit in predicting either the short term (up to seven weeks) or medium term (up to 18 months) prognosis in acute attacks of Crohn's disease treated with oral prednisolone.

This lack of clinical and endoscopic correlation was also shown by Olaison and colleagues (19) who studied the effect of prednisolone treatment (20 to $30 \mathrm{mg}$ daily for six to nine 
weeks) in eight patients with recurrent preanastomotic ileal inflammation. They found a good subjective improvement from glucocorticoid treatment in all patients according to the Harvey and Bradshaw index $(\mathrm{P}<0.01)$. Repeat endoscopy in these patients failed to reveal a corresponding decrease in ileal inflammation.

In the above studies the authors failed to state whether the same observer performed colonoscopies both before and following prednisolone treatment, and they did not detail how observers assessed ulcerations. Sonnenberg et al (20) had already shown that estimation of ulcer size in the upper gastrointestinal tract is very inaccurate even when performed by expert endoscopists. They suggested that measurements of ulcer size at subsequent endoscopies in the same patient should be performed by the same endoscopist to allow more accurate comparison. Because $67 \%$ of the variables in the CDEIS relate to ulcerative lesions, perhaps colonoscopic assessment should urge measurements of ulcer size in the same patient by the same endoscopist.

Colonoscopy postresection: Rutgeerts et al (15) found that the $70 \%$ of patients who had undergone a curative resection had an endoscopic perianastomotic recurrence within one year of surgery. They also showed that severity of the endoscopic recurrence was predictive of the subsequent clinical course; in patients with no lesions or minor lesions (less than five aphthous ulcers), the actuarial rate of symptomatic relapse was $9 \%$ seven years after surgery, whereas patients with more severe endoscopic relapse had a 100\% symptomatic relapse within four years (21). Two more recent studies $(22,23)$ demonstrated endoscopic recurrences as early as six to 12 weeks following surgical resection.

\section{REFERENCES}

1. Elliot PR, Williams CB, Lennard-Jones JE. Colonoscopic diagnosis of minimal change colitis in patients with a normal sigmoidoscopy and normal air-contrast barium enema. Lancet 1982;i:650-1.

2. Waye JD. The role of colonoscopy in the differential diagnosis of inflammatory bowel disease. Gastrointest Endosc 1977;23:150-4.

3. Holdstock G, DuBouley CE, Smith CL. Survey of the use of colonoscopy in inflammatory bowel disease. Dig Dis Sci 1984;8:731-4.

4. Best WR, Becktel JM, Singleton JW. Development of a Crohn's disease activity index. Gastroenterology 1976;70:439-44.

5. Van Hees PAM, Van Elteren PH, Van Lier HJJ. An index of inflammatory activity in patients with Crohn's disease. Gut 1980;21:279-86.

6. Harvey RF, Bradshaw JM. A simple index of Crohn's disease activity. Lancet 1980;i:514.

7. Fielding JF. Management audit in Crohn's disease. Ir J Med Sci 1981;150:162-8.

8. Modigliani R, Mary JY, Simon JF, GETAID. Clinical, biological and endoscopic picture of attacks of Crohn's disease: evolution on prednisolone. Gastroenterology 1990;98:811-8.

9. Lorenze-Meyer H, Malchow H, Miller B, et al. European Cooperative Crohn's Disease Study (ECCDS): Colonoscopy. Digestion 1985;31:109-19.

10. Gomes P, Du Bouley C, Smith CL, Holdstock G. Relationship between disease activity indices and colonoscopic findings in patients with colonic inflammatory bowel disease. Gut 1986;27;92-5.

11. Modigliani R, Mary JY, GETAID. Reproducibility of colonoscopic findings in Crohn's disease: A prospective multicentre study of interobserver variation. Dig Dis Sci 1987;32:1370-9.

12. Mary JY, Modigliani R, GETAID. Development and validation of an endoscopic index of the severity for Crohn's disease: a prospective multicentre study. Gut 1989;30:983-9.

\section{CONCLUSIONS}

Colonoscopy is a useful diagnostic examination in IBD. It is very accurate in detecting disease and assessing disease extent in Crohn's disease. Colonoscopy is useful in detecting postoperative disease recurrence, and the severity of endoscopic recurrence is a useful predictor of the speed of symptomatic relapse (Table 7).

The GETAID group has shown that following an intensive training period, endoscopists can describe colonoscopic findings in a reproducible manner. However, even with training these descriptions were found, in subsequent studies, to correlate poorly with clinical or laboratory indexes of activity and/or short or medium term prognosis. Due to this marked discrepancy, endoscopic findings, although useful in the diagnosis and determination of disease extent, have little or no role to play in the objective follow-up of disease activity in Crohn's disease patients.

If endoscopic findings are to be recorded in multicentre trials, standardization of endoscopic assessment must first be developed by all centres included in the study. Documentation of endoscopic data may be time-consuming, labourintensive and expensive, and may over-burden already heavily used endoscopy suites.

Despite the above limitations and in the absence of a 'gold standard' for measuring disease activity, more accurately defined colonoscopic data should continue to be gathered and analyzed because they may prove to be of value in more objectively monitoring the course of Crohn's disease. Enteroscopic data should also be collected and analyzed to provide similar objectivity in the monitoring of small bowel disease.

13. Landi B, N'Guyen ANH, Cortot A, GETAID. Endoscopic monitoring of Crohn's disease treatment: A prospective, randomized clinical trial. Gastroenterology 1992;102:1647-53.

14. Baron JH, Connell AM, Lennard-Jones JE. Variation between observers in describing mucosal appearances in proctocolitis. BMJ 1964;i:89-92.

15. Rutgeerts P, Geboes K, Vantrappen G, et al. Natural history of recurrent Crohn's disease at the ileocolonic anastomosis after curative surgery. Gut 1984;25:665-72.

16. Holloway H, Mullins W, Fielding JF. Which is the best Crohn's disease activity index for the clinician. Third United European Gastroenterology meeting. Oslo, June 1994. (A1381)

17. Farmer RG, Hawk WA, Turnball RB Jr. Clinical patterns in Crohn's disease: a statistical study of 615 patients. Gastroenterology 1975;68:627-35.

18. Cooke WT, Prior P. Determining disease activity in inflammatory bowel disease. J Clin Gastroenterol 1984;6:17-25.

19. Olaison G, Sjödahl R, Tagesson C. Glucocorticoid treatment in ileal Crohn's disease: relief of symptoms but not of endoscopically viewed inflammation. Gut 1990;31:325-8.

20. Sonnenberg A, Giger M, Kern L, et al. How reliable is determination of ulcer size by endoscopy? BMJ 1979;ii:1322-4.

21. Rutgeerts P, Geboes K, Vantrappen G, et al. Predictability of the postoperative course of Crohn's disease. Gastroenterology 1990;99:956-63.

22. Baert F, Geboes K, Rutgeerts P. Why are recurrent Crohn's lesions after ileal resections always at the ileal side of the anastomosis? Digestive Diseases Week meeting. San Diego, May 1995. (A3528)

23. Lochs H, Fleig NE, Mayer M, et al. Endoscopic recurrence after operative resection in Crohn's disease. Digestive Diseases Week meeting. San Diego, May 1995. (A3529) 


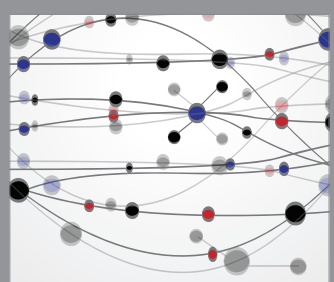

The Scientific World Journal
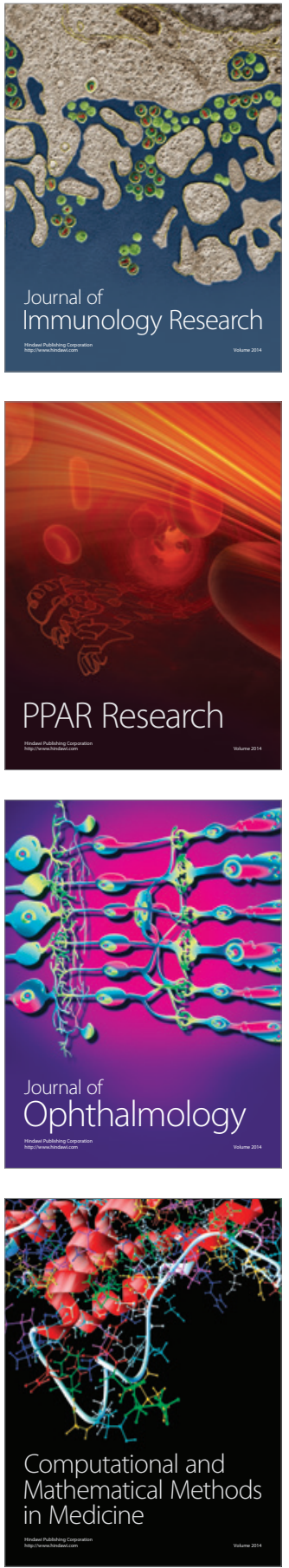

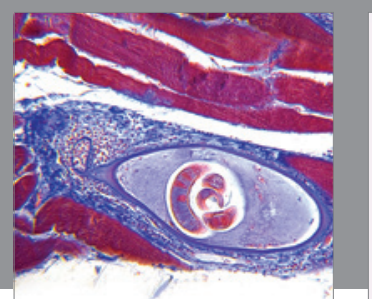

Gastroenterology Research and Practice

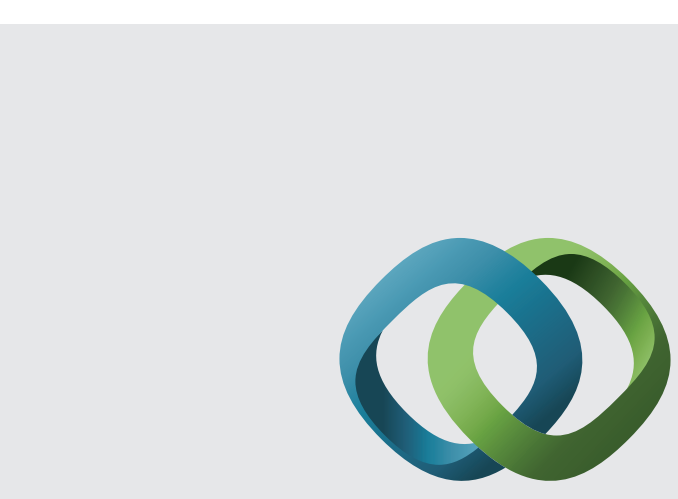

\section{Hindawi}

Submit your manuscripts at

http://www.hindawi.com
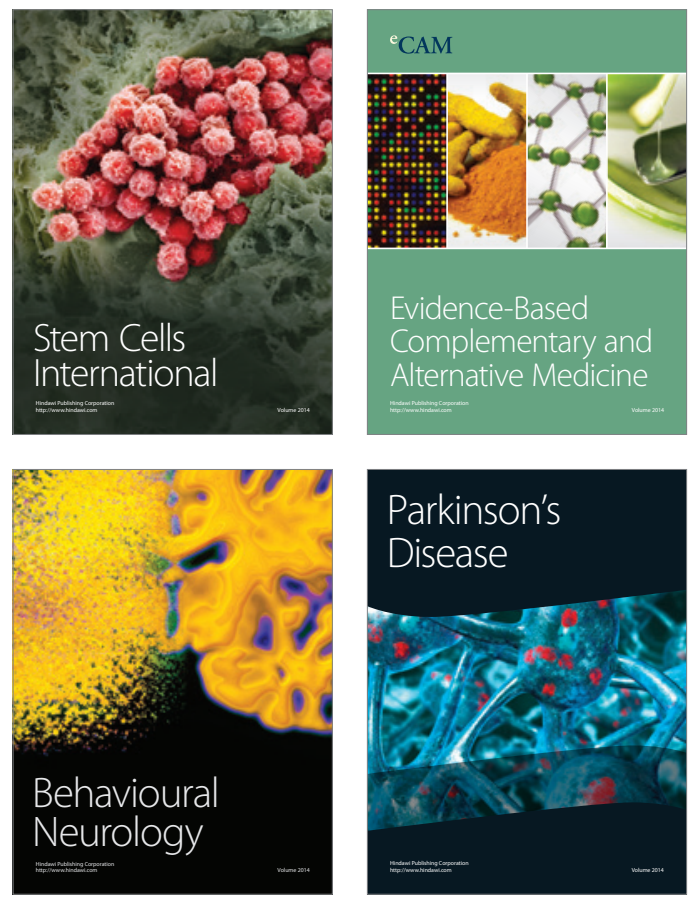
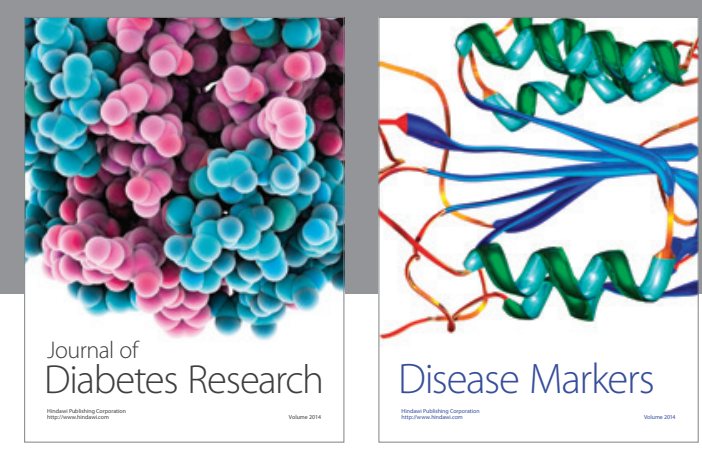

Disease Markers
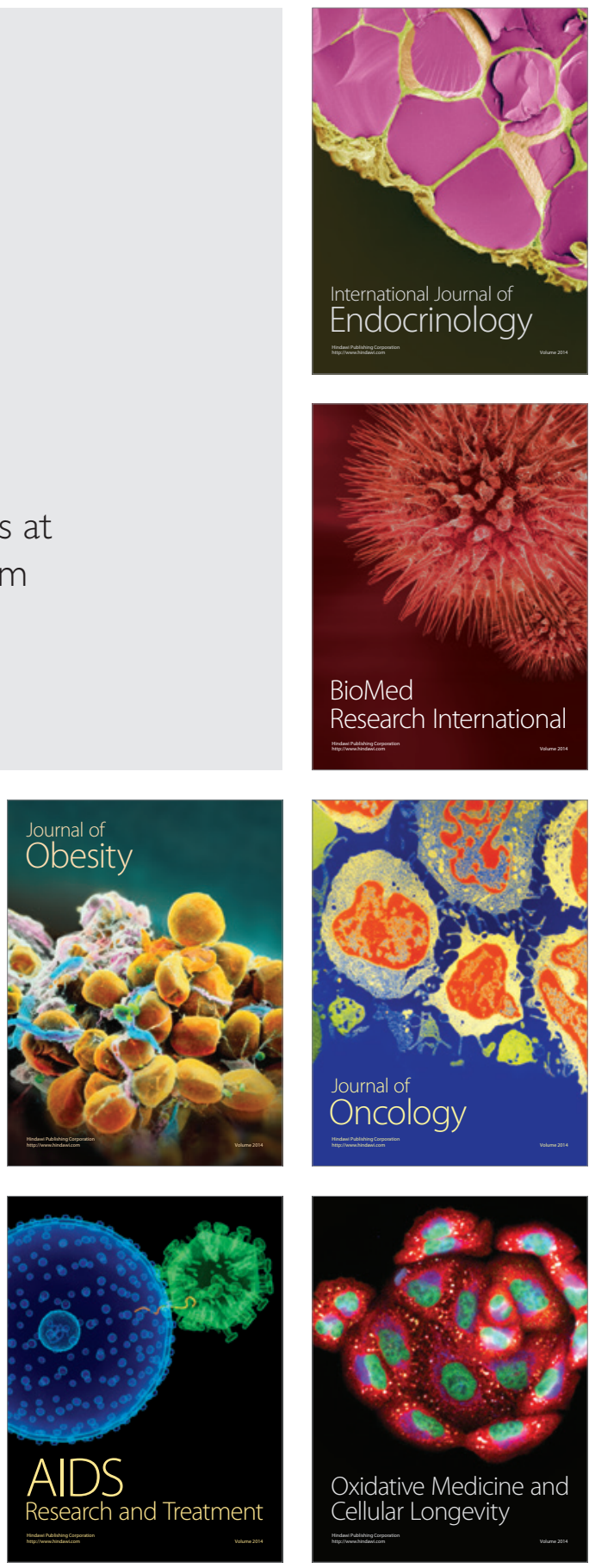